\title{
Leader-Follower Consensus of Linear Multi-Agent Systems With Unknown External Disturbances
}

\author{
Weijun Cao, Jinhui Zhang and Wei Ren
}

\begin{abstract}
This paper addresses the leader-follower consensus tracking problem for multi-agent systems with identical general linear dynamics and unknown external disturbances. First, a distributed extended state observer is proposed, where both the local states and disturbance of each agent are estimated simultaneously by using the relative output information between neighbors. Then a consensus algorithm is proposed for each agent based on the relative estimated states between neighbors and its own disturbance estimate. It is shown that, with the proposed observerbased consensus algorithm, the leader-follower consensus problem can be solved. Finally, we present a simulation example to demonstrate the effectiveness of the proposed algorithm.
\end{abstract}

\section{Index Terms}

Multi-agent systems; Leader-follower consensus; Distributed extended state observer

\section{INTRODUCTION}

In recent years, more and more attention has been paid on cooperative control of multi-agent systems owning to various applications in coordination of unmanned aerial vehicles, cooperation of multi-robot teams, sensor networks, etc. The consensus problem of multi-agent systems, as one important cooperative control problem, is to design a distributed algorithm to make all agents reach an agreement on certain quantities of interest through information exchange between neighbors. Based on the earlier work [1], Olfati-Saber and Murray introduce a theoretical framework for solving the consensus problem for networks of first-order integrators with a fixed or switching topology and communication delays in [2]. For multiagent systems governed by second-order dynamics, the consensus problem is also investigated extensively. It has been shown in [3] that, different from the first-order consensus problem, the second-order consensus might not be achieved even if the network topology has a directed spanning tree. For multi-agent systems with higher-order dynamics, Yu et al. derive some necessary and sufficient conditions for higher-order

Weijun Cao and Jinhui Zhang are with the College of Information Science \& Technology, Beijing University of Chemical Technology, Beijing 100029, China. Wei Ren is with the Department of Electrical Engineering, University of California, Riverside, CA 92521, USA. Email address: jhzhang@mail.buct.edu.cn (J. Zhang); ren@ee.ucr.edu (W. Ren). 
consensus in [4]. It is shown that the higher-order consensus can be reached if and only if all subsystems are asymptotically stable. For more references on first-, second- and higher-order consensus, please refer to $[5]-[8]$, and the references therein.

An interesting topic is the consensus problem of multi-agent systems with a leader, where the leader is followed by all other agents, called the followers, and the motion of the leader is independent of the followers. Such a problem is commonly called the leader-follower consensus problem. In [9], the leaderfollower consensus problem is solved for first-order multi-agent systems in a sampled-data framework, and the consensus convergence analysis is based on the $M$-matrix theory. Hong et al. investigate the second-order leader-follower consensus problem for a multi-agent system with a switching topology in [10], where the velocity of the leader is assumed to be unknown. Recently, the consensus problem of multi-agent systems with general linear dynamics is investigated in [11]-[13]. It is worth noting that most proposed distributed consensus algorithms are based on the relative state information, which is not always available in practical applications. In [14], Zhang et al. study the leader-follower consensus problem of networked systems, where each agent has identical general linear dynamics. Based on the relative output information of neighboring agents, adaptive leaderless and leader-follower consensus algorithms are proposed in [15] for multi-agent systems with general linear dynamics. In [16], a leader-follower consensus problem is considered for multi-agent systems with general linear dynamics and a leader whose control input is nonzero and not available to any follower.

It should be pointed out that, in practical applications, unknown external disturbances are usually unavoidable. Thus, how to solve the consensus problem for multi-agent systems with unknown external disturbances turns out to be an important and interesting issue. In [17], the $H_{\infty}$ consensus problem is investigated for networks of agents with external disturbances and model uncertainties under directed fixed and switching topologies, where the unknown disturbances are assumed to belong to $\mathcal{L}_{2}$. In [18], distributed proportional-integral controllers, which can attenuate constant disturbances in the network, are proposed for first- and second-order integrator systems. In [19], based on disturbance observers, Yang et al. propose some algorithms for second-order multi-agent systems with exogenous disturbances, which can only deal with the case where the disturbances are generated by a linear exogenous system. In [20], Li et al. propose some distributed leader-follower consensus algorithms for linear multi-agent systems subject to different matching uncertainties, where the uncertainties in each agent's dynamics are bounded, and their upper bounds are estimated by adaptive laws.

In this paper, we focus on the leader-follower consensus problem for multi-agent systems with identical general linear dynamics and unknown external disturbances, and the external disturbances are assumed to be unknown but bounded, and have constant steady-state values. The main contributions of this paper 
are listed as follows:

- A distributed extended state observer based on relative output information is designed to estimate the disturbance for each agent. In particular, the relative output information is utilized to determine the disturbance estimate, and the state estimates instead of the actual states themselves are incorporated in the distributed consensus algorithm.

- The distributed controller designed in this paper is continuous, which avoids the chattering phenomenon of the discontinuous controller in [16]. Besides, disturbance estimates are introduced to achieve active rejection of the disturbances.

The rest of this paper is organized as follows. In Section II, some basic knowledge of graph theory and notations are introduced. Section IV studies a leader-follower consensus algorithm that can reject disturbance for identical general linear multi-agent systems. Section V gives some simulation examples to validate our algorithm. Finally, the conclusions are drawn in Section VI.

\section{Preliminaries}

The following notations is used throughout this paper: $\mathbb{R}^{n \times n}$ denotes the set of $n \times n$ real matrices, $I_{n}$ denotes the $n \times n$ identity matrix, 0 denotes a zero matrix with an appropriate dimension. Let $\mathbf{1}_{N}=$ $(1,1, \ldots, 1)_{N}^{T}, \mathbf{0}_{N}=(0,0, \ldots, 0)_{N}^{T}$, and $\operatorname{diag}\left(K_{1}, K_{2}, \ldots, K_{n}\right)$ denote a block-diagonal matrix whose diagonal elements are $K_{1}, K_{2}, \ldots, K_{n}$. For a given real symmetric matrix $W, \lambda_{\max }(W)$ denotes the the largest eigenvalue of $W, \lambda_{\min }(W)$ denotes the the smallest eigenvalue of $W$, and $W<0$ denotes that $W$ is negative definite. $\otimes$ represents the Kronecker product. For matrices $M, N, V, W$, the following properties of the Kronecker product is used in our proof:

$$
\begin{aligned}
& M \otimes(N+V)=M \otimes N+M \otimes V \\
& (M \otimes V)(N \otimes W)=M N \otimes V W \\
& (M \otimes V)^{T}=M^{T} \otimes V^{T} .
\end{aligned}
$$

Consider a multi-agent system consisting of one leader and $N$ followers, the leader is labeled as agent 0 , and the followers are labeled agent 1 to $N$. The communication graph among the $N$ followers is described by a graph $\mathcal{G}=(\mathcal{V}, \mathcal{E})$, where $\mathcal{V}=\{1,2, \ldots, N\}$ is the node set and $\mathcal{E}$ is the edge set. A directed edge $(i, j)$ in a directed graph indicates that agent $i$ can send information to agent $j$ but not necessarily vice versa. Here agent $i$ is a neighbor of agent $j$. An undirected graph is defined such that $(j, i) \in \mathcal{E}$ implies that $(i, j) \in \mathcal{E}$. The adjacency matrix associated with $\mathcal{G}$ is defined as $\mathbb{A}=\left[a_{i j}\right] \in \mathbb{R}^{N \times N}$, where $a_{i j}>0$ if $(j, i) \in \mathcal{E}$, and $a_{i j}=0$ otherwise. For an undirected graph, we assume $a_{j i}=a_{i j}$. The Laplacian matrix associated with $\mathcal{G}$ is defined as $\mathcal{L}=\left[l_{i j}\right] \in \mathbb{R}^{N \times N}$, where $l_{i i}=\sum_{j \neq i} a_{i j}$ and $l_{i j}=-a_{i j}$ for $i \neq j$. 
Zero is an eigenvalue of $\mathcal{L}$ with the corresponding right eigenvector $\mathbf{1}$, and all nonzero eigenvalues have positive real parts. A directed path from node $i$ to node $j$ is a sequence of directed edges in the form $\left\{\left(i, i_{1}\right),\left(i_{1}, i_{2}\right), \ldots,\left(i_{l}, j\right)\right\}$. We assume that the leader does not receive any information from the followers, and the leader is a neighbour of only a part of the followers. Define a matrix $\mathcal{F}=\operatorname{diag}\left(f_{1}, f_{2}, \ldots, f_{N}\right)$. where $f_{i}>0$ if the leader is a neighbor of agent $i$, and $f_{i}=0$ otherwise. Denote the communication graph among the leader and followers as $\hat{\mathcal{G}}$. Define the matrix $\hat{M}=\mathcal{L}+\mathcal{F}$.

\section{Problem Statement}

Consider the multi-agent system composed of one leader and $N$ followers with external disturbances. The dynamics of the followers are denoted by

$$
\begin{aligned}
& \dot{x}_{i}(t)=A x_{i}(t)+B\left[u_{i}(t)+d_{i}(t)\right] \\
& y_{i}(t)=C x_{i}(t), \quad i=1,2, \ldots, N
\end{aligned}
$$

where $x_{i}(t) \in \mathbb{R}^{n}, u_{i}(t) \in \mathbb{R}^{q}, d_{i}(t) \in \mathbb{R}^{q}, y_{i}(t) \in \mathbb{R}^{r}$ are the state, the control input, the external disturbance and the output of the $i$-th follower, respectively, and $A \in \mathbb{R}^{n \times n}, B \in \mathbb{R}^{n \times q}$ and $C \in \mathbb{R}^{r \times n}$. The leader has the following dynamics,

$$
\begin{aligned}
& \dot{x}_{0}(t)=A x_{0}(t) \\
& y_{0}(t)=C x_{0}(t)
\end{aligned}
$$

where $x_{0}(t) \in \mathbb{R}^{n}$ and $y_{0}(t) \in \mathbb{R}^{r}$ are, respectively, the state and the output of the leader.

For system (1), the following assumptions and definition are necessary.

Assumption 1: The follower graph $\mathcal{G}$ is undirected, and the leader has directed paths to all followers.

Assumption 2: The pair $(A, B)$ is controllable, and $A, B, C$ satisfy that

$$
\operatorname{rank}\left[\begin{array}{cc}
C & 0 \\
A & B
\end{array}\right]=n+q .
$$

Assumption 3: The external disturbances for each agent satisfy the conditions: $(1) d_{i}(t)$ and $\dot{d}_{i}(t)$ are bounded; (2) $\lim _{t \rightarrow \infty} \dot{d}_{i}(t)=0$ and $\lim _{t \rightarrow \infty} d_{i}(t)=\omega_{i}$, where $\omega_{i}$ is an unknown constant vector.

Remark 1: The above assumptions have been made on the communication graph, the system matrix, and the external disturbances, respectively.

1) Because of the asymmetry of the Laplacian matrices associated with directed graphs, which leads to significant challenges in designing the distributed consensus algorithm in the presence of unknown external disturbances, we only assume that the follower graph is undirected for simplicity. 
2) In Assumption 2, the condition (2) plays an important role in showing the existence of the distributed extended state observer.

3) In Assumption 3, the disturbances are assumed to be unknown but bounded, and have constant steady-state values. Here, $d_{i}(t)$ represents the lumped disturbance, which could include external disturbances, unmodeled dynamics, and parameter variations. An example can be found in [21], where the disturbance in the MAGnetic LEViation (MAGLEV) suspension system satisfies Assumption 3. For consensus problems of multi-agent systems with other kinds of disturbances, such as harmonic disturbances, it will be an interesting topic for our future research.

For system (1), we introduce the following definition.

Definition 1: The leader-follower consensus problem is solved if

$$
\lim _{t \rightarrow \infty}\left\|x_{i}(t)-x_{0}(t)\right\|=0, \forall i=1,2, \ldots, N .
$$

The objective of this paper is to design an observer-based distributed consensus algorithm for $N$ followers modeled by (1) with unknown disturbances such that the consensus tracking error converges to zero.

\section{Main Results}

By adding an extended state variable $d_{i}(t)$, system (1) can be rewritten into the following extended system form,

$$
\begin{aligned}
\dot{\bar{x}}_{i}(t) & =\bar{A} \bar{x}_{i}(t)+\bar{B} u_{i}(t)+E h_{i}(t) \\
y_{i}(t) & =\bar{C} \bar{x}_{i}(t)
\end{aligned}
$$

where $\bar{x}_{i}(t)=\left[\begin{array}{c}x_{i}(t) \\ d_{i}(t)\end{array}\right], h_{i}(t)=\dot{d}_{i}(t)$ and

$$
\bar{A}=\left[\begin{array}{cc}
A & B \\
0_{q \times n} & 0_{q}
\end{array}\right], \bar{B}=\left[\begin{array}{c}
B \\
0_{q}
\end{array}\right], E=\left[\begin{array}{c}
0_{n \times q} \\
I_{q}
\end{array}\right], \bar{C}=\left[\begin{array}{cc}
C & 0_{r \times q}
\end{array}\right] .
$$

Motivated by the extended state observer technique for single systems proposed in [22], we introduce the following distributed extended state observer based on relative output information for each follower:

$$
\dot{\overline{\hat{x}}}_{i}(t)=\bar{A} \hat{\bar{x}}_{i}(t)+\bar{B} u_{i}(t)+\nu_{i}(t)
$$

where $\hat{\bar{x}}_{i}(t)=\left[\begin{array}{c}\hat{x}_{i}(t) \\ \hat{d}_{i}(t)\end{array}\right], \hat{x}_{i}(t)$ is the state estimate of the $i$-th agent, $\hat{d}_{i}(t)$ is the disturbance estimate, and

$$
\nu_{i}(t)=F\left(\sum_{j=1}^{N} a_{i j}\left[\tilde{y}_{i}(t)-\tilde{y}_{j}(t)\right]+f_{i} \tilde{y}_{i}(t)\right)
$$


with $\tilde{y}_{i}(t)=y_{i}(t)-C \hat{x}_{i}(t), F \in \mathbb{R}^{(n+q) \times r}$ being the observer gain matrix to be designed, and $a_{i j}$ and $f_{i}$ being defined in Section II. Then, the following distributed consensus algorithm is proposed for each follower,

$$
u_{i}(t)=K \sum_{j=1}^{N} a_{i j}\left[\hat{x}_{i}(t)-\hat{x}_{j}(t)\right]+K f_{i}\left[\hat{x}_{i}(t)-x_{0}(t)\right]-\hat{d}_{i}(t)
$$

where $K \in \mathbb{R}^{q \times n}$ is the feedback gain matrix to be determined later.

Remark 2: It follows from (4) that $\dot{\hat{d}}_{i}(t)=\left[\begin{array}{ll}0 & I_{q}\end{array}\right] \nu_{i}(t)$. Thus, the disturbance estimate $\hat{d}_{i}(t)$ is essentially an integral of a part of $\nu_{i}(t)$. Thus the consensus algorithm (5) can be seen as a PI-like one in the context of output feedback instead of state feedback. In [18], a distributed PI consensus algorithm is proposed to attenuate the constant disturbance for double-integrator dynamics. The algorithm (5) distinguishes from the algorithm in [18] in three aspects, namely, 1) the algorithm (5) is established for agents with general linear dynamics, 2) the relative output information is utilized to determine the disturbance estimate $\left.\hat{d}_{i}(t), 3\right)$ the state estimates instead of the states themselves are incorporated in the algorithm (5).

Remark 3: In the related works [15], [16], distributed consensus controllers are presented for multiagent systems with a leader whose control input is bounded and nonzero. While these references allow for more general leader states, it is worth mentioning that the controllers are discontinuous, and the chattering phenomenon is inevitable. Compared with [15], [16], the main features of the presented consensus algorithm are at least twofold. First, the controller (5) is continuous. Second, in [15], [16], the effects of the unknown control input in the leader are attenuated by the discontinuous function, and thus the approaches in [15], [16] are disturbance attenuation-based ones. However, in the proposed algorithm (5), the disturbance estimates are introduced to eliminate the influence of the disturbances from the state equations in steady state, which is an active disturbance rejection-based method.

Define $x(t)=\left[x_{1}^{T}(t), \ldots, x_{N}^{T}(t)\right]^{T}$, and $d(t)=\left[d_{1}^{T}(t), \ldots, d_{N}^{T}(t)\right]^{T}$. Also define the state and disturbance estimation errors as, respectively,

$$
\begin{aligned}
& e_{x}(t)=\hat{x}(t)-x(t), \\
& e_{d}(t)=\hat{d}(t)-d(t) .
\end{aligned}
$$

Combining (3), (4), (6) and (7), the estimation error equation can be written as:

$$
\dot{e}(t)=\left(I_{N} \otimes \bar{A}+\hat{M} \otimes F \bar{C}\right) e(t)-\left(I_{N} \otimes E\right) h(t)
$$

where $e(t) \triangleq\left[\begin{array}{l}e_{x}(t) \\ e_{d}(t)\end{array}\right], h(t) \triangleq\left[h_{1}^{T}(t), \ldots, h_{N}^{T}(t)\right]^{T}$. 
Lemma 1: The pair $(\bar{A}, \bar{C})$ is observable if and only if $(A, C)$ is observable and Condition (2) is satisfied.

Proof: From the well-known PBH rank test for observability, the pair $(\bar{A}, \bar{C})$ is observable if and only if the following condition is satisfied:

$$
\operatorname{rank}\left[\begin{array}{c}
\lambda I_{n+q}-\bar{A} \\
\bar{C}
\end{array}\right]=n+q, \forall \lambda \in \mathbb{C} \text {. }
$$

Note that

$$
\operatorname{rank}\left[\begin{array}{c}
\lambda I_{n+q}-\bar{A} \\
\bar{C}
\end{array}\right]=\operatorname{rank}\left[\begin{array}{cc}
C & 0 \\
\lambda I_{n}-A & -B \\
0 & \lambda I_{q}
\end{array}\right] .
$$

Thus, i) if $\lambda \neq 0$, then

$$
\operatorname{rank}\left[\begin{array}{c}
\lambda I_{n+q}-\bar{A} \\
\bar{C}
\end{array}\right]=\operatorname{rank}\left[\begin{array}{c}
C \\
\lambda I_{n}-A
\end{array}\right]+q
$$

which together with the fact that $(A, C)$ is observable gives (9);

ii) if $\lambda=0$, then

$$
\operatorname{rank}\left[\begin{array}{c}
\lambda I_{n+q}-\bar{A} \\
\bar{C}
\end{array}\right]=\operatorname{rank}\left[\begin{array}{cc}
C & 0 \\
-A & -B
\end{array}\right]
$$

which also implies that equation (9) holds under Condition 2.

Before presenting the main results of this paper, we first recall the following useful lemma.

Lemma 2: [23], [24] Under Assumption 1, the matrix $\hat{M}$ is symmetric positive definite.

The following lemma plays an important role in deriving the main results.

Lemma 3: For given gain matrices $K$ and $F$ and the diagonal matrix $\Delta=\operatorname{diag}\left\{\delta_{1}, \delta_{2}, \ldots, \delta_{N}\right\}$ with $0<\delta_{1} \leq \delta_{2} \leq \cdots \leq \delta_{N}$, the following two statements are equivalent.

(S1) There exists a symmetric positive-definite matrix $P \triangleq\left[\begin{array}{cc}P_{11} & P_{12} \\ P_{12}^{T} & P_{22}\end{array}\right], P_{11} \in \mathbb{R}^{n \times n}, P_{12} \in \mathbb{R}^{n \times(n+q)}$, $P_{22} \in \mathbb{R}^{(n+q) \times(n+q)}$, satisfying the following conditions:

$$
\mathcal{A}^{T} \mathcal{P}+\mathcal{P} \mathcal{A}<0
$$

where

$$
\begin{aligned}
& \mathcal{A}=\left[\begin{array}{cc}
I_{N} \otimes A+\Delta \otimes B K & {\left[\begin{array}{cc}
\Delta \otimes B K & -I_{N} \otimes B
\end{array}\right]} \\
0 & I_{N} \otimes \bar{A}+\Delta \otimes F \bar{C}
\end{array}\right], \\
& \mathcal{B}=\left[\begin{array}{c}
0 \\
-I_{N} \otimes E
\end{array}\right], \mathcal{P} \triangleq\left[\begin{array}{cc}
I_{N} \otimes P_{11} & I_{N} \otimes P_{12} \\
I_{N} \otimes P_{12}^{T} & I_{N} \otimes P_{22}
\end{array}\right] .
\end{aligned}
$$


(S2) There exists symmetric positive-definite matrices $P_{1}$ and $P_{2}$ with appropriate dimensions satisfying the following conditions for $i=1,2, \ldots, N$,

$$
\begin{gathered}
\left(A+\delta_{i} B K\right)^{T} P_{1}+P_{1}\left(A+\delta_{i} B K\right)<0, \\
\left(\bar{A}+\delta_{i} F \bar{C}\right)^{T} P_{2}+P_{2}\left(\bar{A}+\delta_{i} F \bar{C}\right)<0 .
\end{gathered}
$$

Proof: $(\mathrm{S} 1) \Rightarrow(\mathrm{S} 2)$ : Suppose that there exists a matrix $P>0$ that satisfies Condition (10). It is easy to show that there exists an elementary transformation matrix $\mathcal{I}_{2 n N}$ satisfying $\mathcal{I}_{2 n N}\left(I_{N} \otimes P\right) \mathcal{I}_{2 n N}=\mathcal{P}$. Thus, $\mathcal{P}>0$ if and only if $P>0$.

Letting $\mathcal{A}_{11}=I_{N} \otimes A+\Delta \otimes B K, \mathcal{A}_{12}=\left[\Delta \otimes B K \quad-I_{N} \otimes B\right], \mathcal{A}_{22}=I_{N} \otimes \bar{A}+\Delta \otimes F \bar{C}$ and substituting $\mathcal{P}$ into (10), we can get:

$$
\left[\begin{array}{ll}
\Gamma_{(1,1)} & \Gamma_{(1,2)} \\
\Gamma_{(1,2)}^{T} & \Gamma_{(2,2)}
\end{array}\right]<0
$$

where $\Gamma_{(1,1)}=I_{N} \otimes\left(A^{T} P_{11}+P_{11} A\right)+\Delta \otimes\left(K^{T} B^{T} P_{11}+P_{11} B K\right), \Gamma_{(1,2)}=\left(I_{N} \otimes P_{11}\right) \mathcal{A}_{12}+\left(I_{N} \otimes\right.$ $\left.P_{12}\right) \mathcal{A}_{22}+\mathcal{A}_{11}^{T}\left(I_{N} \otimes P_{12}\right), \Gamma_{(2,2)}=\left(I_{N} \otimes P_{12}^{T}\right) \mathcal{A}_{12}+\left(I_{N} \otimes P_{22}\right) \mathcal{A}_{22}+\mathcal{A}_{12}^{T}\left(I_{N} \otimes P_{12}\right)+\mathcal{A}_{22}^{T}\left(I_{N} \otimes P_{22}\right)$. Let $P_{1}=P_{11}$. It follows from (13) that $\Gamma_{(1,1)}<0$, which further implies that Condition (11) is satisfied. Next, we define $P^{-1}=\left[\begin{array}{cc}S_{11} & S_{12} \\ S_{12}^{T} & S_{22}\end{array}\right]$ and $\mathcal{S} \triangleq\left[\begin{array}{cc}I_{N} \otimes S_{11} & I_{N} \otimes S_{12} \\ I_{N} \otimes S_{12}^{T} & I_{N} \otimes S_{22}\end{array}\right]$. It is easy to verify that $\mathcal{S}=\mathcal{P}^{-1}$. Pre- and post-multiplying $(10)$ by $\mathcal{S}$, we have

$$
\mathcal{S} \mathcal{A}^{T}+\mathcal{A S}<0
$$

Substituting $\mathcal{A}$ and $\mathcal{S}$ into (14) gives

$$
\left[\begin{array}{ll}
\Sigma_{(1,1)} & \Sigma_{(1,2)} \\
\Sigma_{(1,2)}^{T} & \Sigma_{(2,2)}
\end{array}\right]<0
$$

where $\Sigma_{(1,1)}=\left(I_{N} \otimes S_{11}\right) \mathcal{A}_{11}^{T}+\left(I_{N} \otimes S_{12}\right) \mathcal{A}_{12}^{T}+\mathcal{A}_{11}\left(I_{N} \otimes S_{11}\right)+\mathcal{A}_{12}\left(I_{N} \otimes S_{12}^{T}\right), \Sigma_{(1,2)}=\left(I_{N} \otimes S_{12}\right) \mathcal{A}_{22}^{T}+$ $\mathcal{A}_{11}\left(I_{N} \otimes S_{12}\right)+\mathcal{A}_{12}\left(I_{N} \otimes S_{22}\right), \Sigma_{(2,2)}=I_{N} \otimes\left(\bar{A} S_{22}+S_{22} \bar{A}^{T}\right)+\Delta \otimes\left(F \bar{C} S_{22}+S_{22} \bar{C}^{T} F^{T}\right)$. Let $P_{2}=S_{22}^{-1}$. It follows from (15) that $\Sigma_{(2,2)}<0$, which further implies that Condition (12) is satisfied.

$(\mathrm{S} 2) \Rightarrow(\mathrm{S} 1)$ : According to $(11)$ and $(12)$, it is easy to obtain that

$$
\begin{aligned}
& \Upsilon_{1} \triangleq \mathcal{A}_{11}^{T}\left(I_{N} \otimes P_{1}\right)+\left(I_{N} \otimes P_{1}\right) \mathcal{A}_{11}<0 \\
& \Upsilon_{2} \triangleq \mathcal{A}_{22}^{T}\left(I_{N} \otimes P_{2}\right)+\left(I_{N} \otimes P_{2}\right) \mathcal{A}_{22}<0
\end{aligned}
$$

By using the Schur complement lemma, it is easy to see that there exists a scalar $\epsilon_{0}$ such that

$$
\left[\begin{array}{ll}
\Upsilon_{1} & \Psi^{T} \\
\Psi & \epsilon \Upsilon_{2}
\end{array}\right]<0
$$


for all $\epsilon \geq \epsilon_{0}$, where $\Psi=\mathcal{A}_{12}^{T}\left(I_{N} \otimes P_{1}\right)$. Here, we choose $\mathcal{P}$ as

$$
\mathcal{P}=\left[\begin{array}{cc}
I_{N} \otimes P_{1} & 0 \\
0 & \epsilon\left(I_{N} \otimes P_{2}\right)
\end{array}\right]
$$

Then it can be concluded that (16) is equivalent to (10). This completes the proof.

To present our main result, we recall the following lemma.

Lemma 4: [25] Consider the following linear system

$$
\dot{x}(t)=A x(t)+B u(t) .
$$

If $A$ is a Hurwitz matrix and $u(t)$ is bounded and satisfies $\lim _{t \rightarrow \infty} u(t)=0$, then $\lim _{t \rightarrow \infty} x(t)=0$. Then, we have the following result.

Theorem 1: Suppose that Assumptions 1-3 are satisfied. For (1), the leader-follower consensus problem is solved if $K=-\tau B^{T} P_{1}, F=-\tau P_{2}^{-1} \bar{C}^{T}$, and $P_{1}$ and $P_{2}$ are, respectively, the solutions of the following two matrix inequalities:

$$
\begin{aligned}
& A^{T} P_{1}+P_{1} A-2 P_{1} B B^{T} P_{1}<0, \\
& \bar{A}^{T} P_{2}+P_{2} \bar{A}-2 \bar{C}^{T} \bar{C}<0,
\end{aligned}
$$

where $\tau \geq \frac{1}{\lambda_{\min }(\hat{M})}$ is a positive constant.

Proof: Under Assumption 2, it follows from Lemma 1 that Observer (4) exists. Define $e_{d i}(t)=$ $\hat{d}_{i}(t)-d_{i}(t)$. Then the closed-loop network dynamics using (5) for (1) can be written as:

$$
\begin{aligned}
\dot{x}_{i}(t)= & A x_{i}(t)+B K \sum_{j=1}^{N} a_{i j}\left(\hat{x}_{i}(t)-\hat{x}_{j}(t)\right) \\
& +B K f_{i}\left(\hat{x}_{i}(t)-x_{0}(t)\right)-B e_{d i}(t) .
\end{aligned}
$$

Define the consensus tracking error between follower $i$ and the leader as $\varepsilon_{i}(t)=x_{i}(t)-x_{0}(t)$. Let $e_{x i}(t)=\hat{x}_{i}(t)-x_{i}(t)$. We have

$$
\begin{aligned}
\dot{\varepsilon}_{i}(t)= & A \varepsilon_{i}(t)+B K\left(\sum_{j=1}^{N} a_{i j}\left[\varepsilon_{i}(t)-\varepsilon_{j}(t)+e_{x i}(t)-e_{x j}(t)\right]\right. \\
& \left.+f_{i}\left[\varepsilon_{i}(t)+e_{x i}(t)\right]\right)-B e_{d i}(t) .
\end{aligned}
$$

Letting $\varepsilon(t)=\left[\varepsilon_{1}^{T}(t), \varepsilon_{2}^{T}(t), \ldots, \varepsilon_{N}^{T}(t)\right]^{T},(19)$ can be rewritten in a compact form as

$$
\begin{aligned}
\dot{\varepsilon}(t)= & \left(I_{N} \otimes A+\hat{M} \otimes B K\right) \varepsilon(t)+(\hat{M} \otimes B K) e_{x}(t) \\
& -\left(I_{N} \otimes B\right) e_{d}(t) .
\end{aligned}
$$


Combining (20) with the estimation error system (8) gives the closed-loop network system in the following compact form,

$$
\begin{aligned}
{\left[\begin{array}{c}
\dot{\varepsilon}(t) \\
\dot{e}(t)
\end{array}\right]=} & {\left[\begin{array}{cc}
I_{N} \otimes A+\hat{M} \otimes B K & {\left[\begin{array}{cc}
\hat{M} \otimes B K & -I_{N} \otimes B \\
0 & I_{N} \otimes \bar{A}+\hat{M} \otimes F \bar{C}
\end{array}\right]} \\
& +\left[\begin{array}{c}
0 \\
-I_{N} \otimes E
\end{array}\right] h(t) .
\end{array}\right.} \\
& {\left[\begin{array}{c}
\varepsilon(t) \\
e(t)
\end{array}\right] }
\end{aligned}
$$

Using Assumption 1, it follows from Lemma 2 that $\hat{M}$ is symmetric positive definite. There exists an orthogonal matrix $U \in \mathbb{R}^{N \times N}$ satisfying:

$$
U^{T} \hat{M} U=\operatorname{diag}\left(\hat{\lambda}_{1}, \hat{\lambda}_{2}, \ldots, \hat{\lambda}_{N}\right)=\hat{\Delta}
$$

where $0<\hat{\lambda}_{1} \leq \hat{\lambda}_{2} \leq \cdots \leq \hat{\lambda}_{N}$. Take the state transformation

$$
\begin{aligned}
& \bar{\varepsilon}(t) \triangleq\left(U^{T} \otimes I_{n}\right) \varepsilon(t), \bar{e}(t) \triangleq\left(U^{T} \otimes I_{2 n}\right) e(t), \\
& \bar{d}(t) \triangleq\left(U^{T} \otimes I_{q}\right) d(t), \bar{h}(t) \triangleq\left(U^{T} \otimes I_{q}\right) h(t),
\end{aligned}
$$

and define $\eta(t)=\left[\bar{\varepsilon}^{T}(t), \bar{e}^{T}(t)\right]^{T}$, and

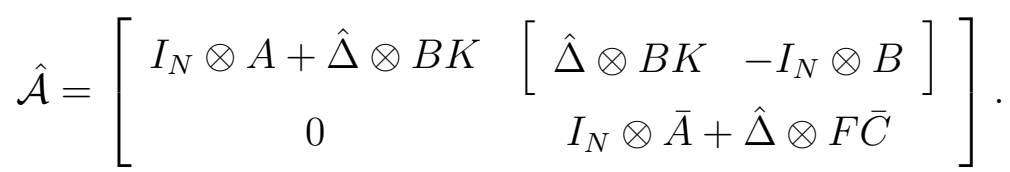

The system (21) can be reformulated as

$$
\dot{\eta}(t)=\hat{\mathcal{A}} \eta(t)+\mathcal{B} \bar{h}(t)
$$

By taking $K=-\tau B^{T} P_{1}, F=-\tau P_{2}^{-1} \bar{C}^{T}$ with $\tau \geq \frac{1}{\lambda_{\min }(\hat{M})}$ and notice $0<\lambda_{\min }(\hat{M})=\hat{\lambda}_{1} \leq \hat{\lambda}_{2} \leq$ $\cdots \leq \hat{\lambda}_{N}$. It is obvious that (17) and (18) imply that the following two inequalities hold for $i=1,2, \ldots, N$, respectively,

$$
\begin{gathered}
\left(A+\hat{\lambda}_{i} B K\right)^{T} P_{1}+P_{1}\left(A+\hat{\lambda}_{i} B K\right)<0, \\
\left(\bar{A}+\hat{\lambda}_{i} F \bar{C}\right)^{T} P_{2}+P_{2}\left(\bar{A}+\hat{\lambda}_{i} F \bar{C}\right)<0 .
\end{gathered}
$$

According to Lemma 3, (22) and (23) hold if and only if the following condition is satisfied,

$$
\hat{\mathcal{A}}^{T} \mathcal{P}+\mathcal{P} \hat{\mathcal{A}}<0
$$

which is equivalent to that $\hat{\mathcal{A}}$ is Hurwitz according to the well-known Lyapunov stability theorem. Under Assumption 3, $\lim _{t \rightarrow \infty} h(t)=0$, it is obvious to note that $\lim _{t \rightarrow \infty}\|\bar{h}(t)\|=0$. Then, it follows from Lemma 4 that $\varepsilon(t) \rightarrow 0$, which indicates that the consensus tracking error converges to zero, and the proof is completed. 
Remark 4: In algorithm (5), the disturbance estimates $\hat{d}_{i}(t)$ are introduced to actively reject the disturbance in each follower. If we choose the following consensus algorithm without considering the disturbance rejection as in [14],

$$
u_{i}(t)=K \sum_{j=1}^{N} a_{i j}\left[\hat{x}_{i}(t)-\hat{x}_{j}(t)\right]+K f_{i}\left[\hat{x}_{i}(t)-x_{0}(t)\right]
$$

it is easy to obtain the estimation error system as

$$
\dot{e}_{x}(t)=\left(I_{N} \otimes A+\hat{M} \otimes F C\right) e_{x}(t)-\left(I_{N} \otimes B\right) d(t)
$$

and the consensus error dynamics can be determined as

$$
\dot{\varepsilon}(t)=\left(I_{N} \otimes A+\hat{M} \otimes B K\right) \varepsilon(t)+(\hat{M} \otimes B K) e_{x}(t)+\left(I_{N} \otimes B\right) d(t) .
$$

It should be noted from (26) and (27) that, due to the perturbation terms $-\left(I_{N} \otimes B\right) d(t)$ and $\left(I_{N} \otimes B\right) d(t)$ in, respectively, (26) and (27), a nonzero disturbance $d(t)$ would result in a nonzero consensus tracking error $\varepsilon(t)$. When all $d_{i}(t)$ satisfy the conditions in Assumption 3, unlike our proposed algorithm, using (25) will lead to a nonzero consensus tracking error. Thus, using (25), the leader-follower consensus problem cannot be solved in the presence of unknown external disturbances.

Remark 5: In the consensus algorithm (5), the disturbance estimate $\hat{d}_{i}(t)$ is incorporated to actively compensate for the effect of the unknown disturbance, which enables the proposed consensus algorithm to be robust with respect to the disturbance satisfying Assumption 3.

\section{Simulation Example}

In this section, a simulation example is provided to validate the effectiveness of our algorithm. Consider a network of five agents, with states $x_{i}(t)=\left[\begin{array}{l}x_{1, i}(t) \\ x_{2, i}(t)\end{array}\right], i=1,2, \ldots, 5$. Their system matrices are

$$
A=\left[\begin{array}{cc}
0 & 1 \\
-1 & 0
\end{array}\right], B=\left[\begin{array}{l}
1 \\
1
\end{array}\right], C=\left[\begin{array}{ll}
1 & 0
\end{array}\right]
$$


and the disturbances associated with each agent are

$$
\begin{aligned}
& d_{1}(t)= \begin{cases}1+\mathrm{e}^{-0.001 t} x_{1,1}(t), & \text { if } t<25 \mathrm{~s} \\
2+\mathrm{e}^{-0.001(t-25)} x_{2,1}(t), & \text { if } t \geq 25 \mathrm{~s}\end{cases} \\
& d_{2}(t)= \begin{cases}2-1.5 \mathrm{e}^{-0.0005 t} x_{1,2}(t), & \text { if } t<25 \mathrm{~s} \\
-4+1.5 \mathrm{e}^{-0.0008(t-25)} x_{2,2}(t), & \text { if } t \geq 25 \mathrm{~s}\end{cases} \\
& d_{3}(t)= \begin{cases}-1+2 \mathrm{e}^{-0.002 t} x_{1,3}(t), & \text { if } t<25 \mathrm{~s} \\
8+2 \mathrm{e}^{-0.001(t-25)} x_{2,3}(t), & \text { if } t \geq 25 \mathrm{~s}\end{cases} \\
& d_{4}(t)= \begin{cases}4-2.5 \mathrm{e}^{-0.001 t} x_{1,4}(t), & \text { if } t<25 \mathrm{~s} \\
-6-2.8 \mathrm{e}^{-0.001(t-25)} x_{2,4}(t), & \text { if } t \geq 25 \mathrm{~s}\end{cases} \\
& d_{5}(t)= \begin{cases}5-3 \mathrm{e}^{-0.001 t} x_{1,5}(t), & \text { if } t<25 \mathrm{~s} \\
-10-5 \mathrm{e}^{-0.001(t-25)} x_{2,5}(t), & \text { if } t \geq 25 \mathrm{~s} .\end{cases}
\end{aligned}
$$

Assume that the communication graph is given by Fig. 1. We let $a_{i j}=1$ if $j$ is a neighbor of $i$. Here the leader is a neighbour of the fourth and fifth followers with $f_{4}=f_{5}=1$ and $f_{i}=0$ otherwise. The matrix $\hat{M}$ can be determined as

$$
\hat{M}=\left[\begin{array}{ccccc}
2 & 0 & -1 & 0 & -1 \\
0 & 3 & -1 & -1 & -1 \\
-1 & -1 & 3 & -1 & 0 \\
0 & -1 & -1 & 4 & -1 \\
-1 & -1 & 0 & -1 & 4
\end{array}\right]
$$

with eigenvalues $0.3292,2.1383,3.4480,4.5028,5.5817$.

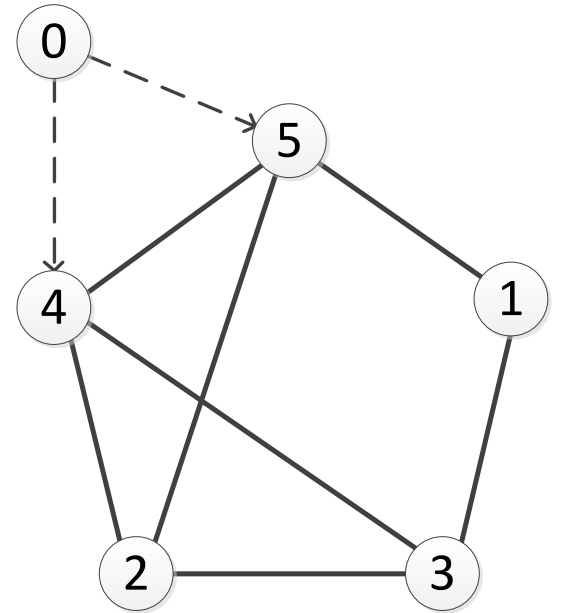

Fig. 1. The communication topology 
For simplicity, we choose $\tau=\frac{1}{0.3292}=3.038$. By solving (11) and (12), we get the solutions $P_{1}$ and $P_{2}$ as follows,

$$
P_{1}=\left[\begin{array}{cc}
0.4654 & 0 \\
0 & 0.2668
\end{array}\right], P_{2}=\left[\begin{array}{ccc}
0.8988 & -0.3999 & -0.3745 \\
-0.3999 & 0.7490 & -0.3996 \\
-0.3745 & -0.3996 & 1.1984
\end{array}\right] \text {. }
$$

According to Theorem 1, the gain matrices $K$ and $F$ can be computed as

$$
\begin{aligned}
& K=-\tau B^{T} P_{1}=\left[\begin{array}{ll}
-1.4139 & -0.8104
\end{array}\right], \\
& F=-\tau P_{2}^{-1} \bar{C}^{T}=\left[\begin{array}{l}
9.0825 \\
7.7408 \\
5.4193
\end{array}\right] .
\end{aligned}
$$

The state trajectories of the five agents using algorithm (5) are depicted in Figs. 2(a) and 2(b). The disturbances and their estimates for each agent are given in Fig. 3. It can be seen that, with the proposed algorithm (5), the leader-follower consensus of the multi-agent systems can be achieved in the presence of external disturbances. To illustrate the advantages of the proposed distributed consensus algorithm (5), we consider the algorithm (25) proposed in [14], where $K=-c R^{-1} B^{T} P$, with $c \geq \frac{1}{2 \lambda_{\min }(\hat{M})}$ and $P$ is the unique positive definite solution of the following algebraic Riccati equation:

$$
A^{T} P+P A+Q-P B R^{-1} B^{T} P=0 .
$$

Let $c=\frac{1}{2 \times 0.3292}=1.5189$. Choosing $R=1$ and solving the algebraic Riccati equation (28), we have:

$$
P=\left[\begin{array}{cc}
1.6373 & -0.3406 \\
-0.3406 & 0.9052
\end{array}\right], K=\left[\begin{array}{ll}
-1.2966 & -0.5646
\end{array}\right] \text {. }
$$

The state trajectories of the five agents using (25) are depicted in Figs. 2(c) and 2(d). It can be found that the leader-follower consensus cannot be reached in this case.

\section{CONCLUSIONS}

The leader-follower consensus problem is investigated for multi-agent systems with general linear dynamics and unknown external disturbances. The consensus algorithm is proposed based on state and disturbance estimates, which are achieved by a distributed extend state observer using relative output information. With the proposed algorithm and observer, the disturbances are rejected in each agent actively, and the consensus tracking errors are cooperatively uniformly ultimately bounded and converge to an adjustable neighborhood of the origin. Finally, a numerical example is given to illustrate the theoretical analysis. 


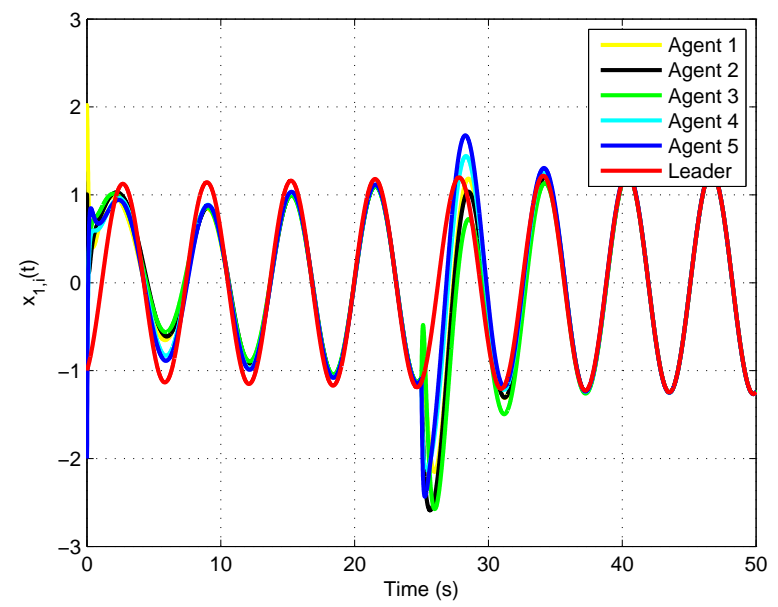

(a) States $x_{1, i}(t)$ with consensus algorithm (5)

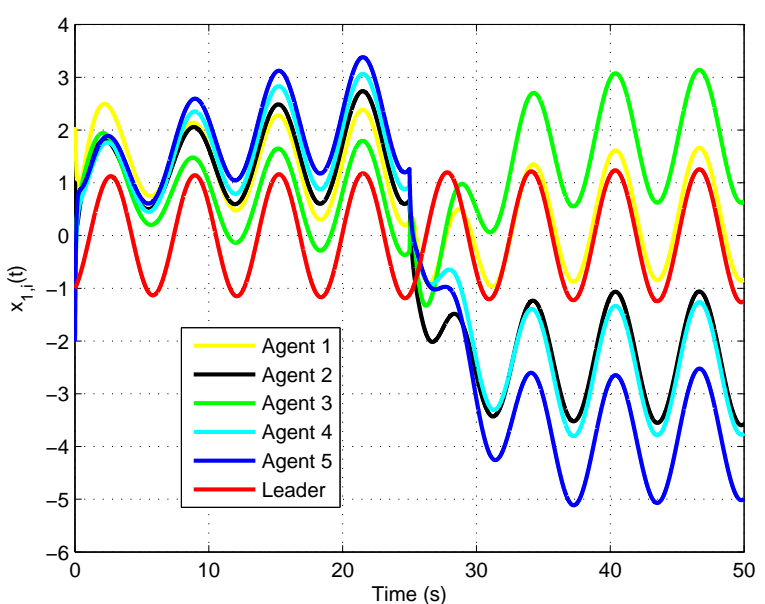

(c) States $x_{1, i}(t)$ with consensus algorithm (25)

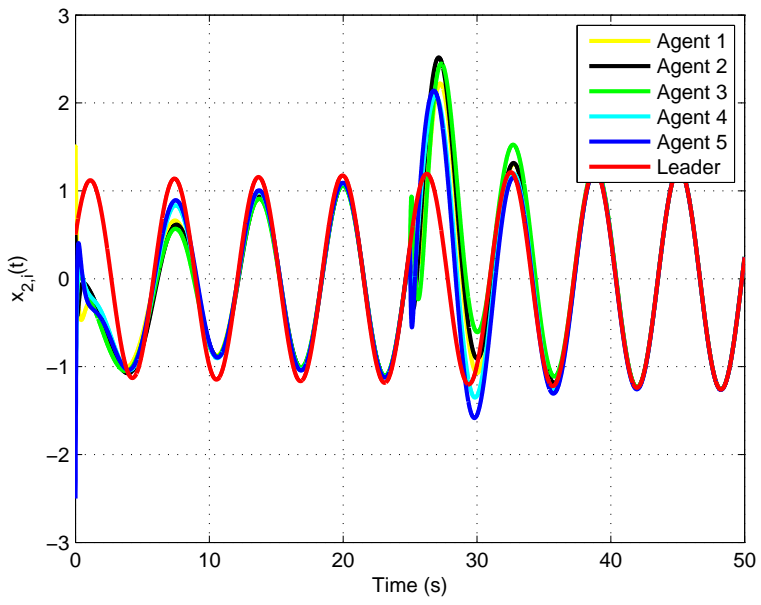

(b) States $x_{2, i}(t)$ with consensus algorithm (5)

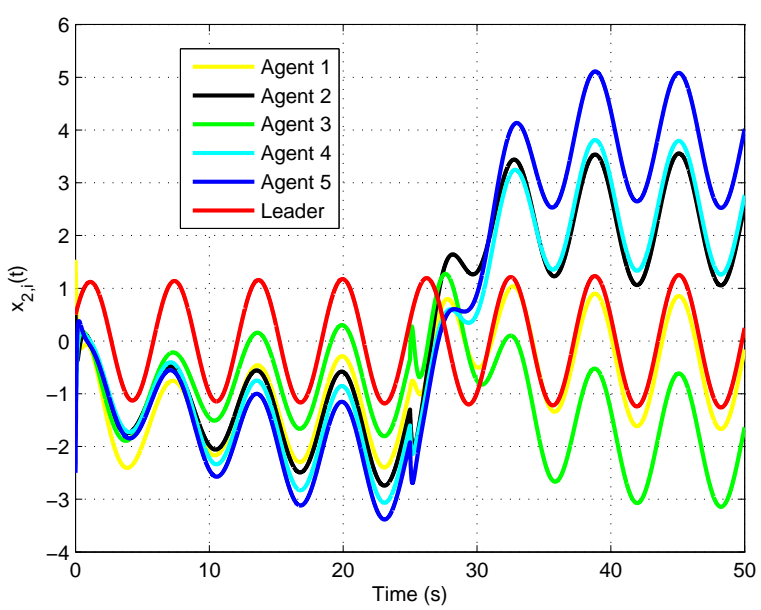

(d) States $x_{2, i}(t)$ with consensus algorithm (25)

Fig. 2. State trajectories of the five agents

\section{ACKNOWLEGEMENTS}

The authors wish to thank the associate editor and referees for their very constructive comments and suggestions, which have helped improve the presentation of the paper. This work was partially supported by the National Natural Science Foundation of China 61473024 and 61403018.

\section{REFERENCES}

[1] A. Jadbabaie, J. Lin, and A. S. Morse, "Coordination of groups of mobile autonomous agents using nearest neighbor rules," IEEE Trans. Autom. Control, vol. 48, no. 6, pp. 988-1001, 2003.

[2] R. Olfati-Saber and R. Murray, "Consensus problems in networks of agents with switching topology and time delays," IEEE Trans. Autom. Control, vol. 49, no. 9, pp. 1520-1533, 2004.

[3] W. Ren and E. Atkins, "Distributed multi-vehicle coordinated control via local information exchange," Int. J. Robust and Nonlinear Control, vol. 17, no. 10-11, pp. 1002-1033, 2007. 


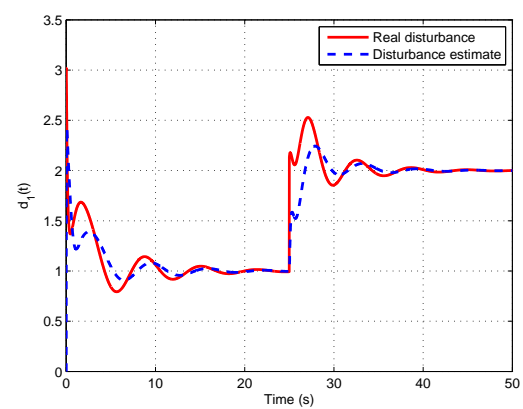

(a) $d_{1}(t)$ and $\hat{d}_{1}(t)$

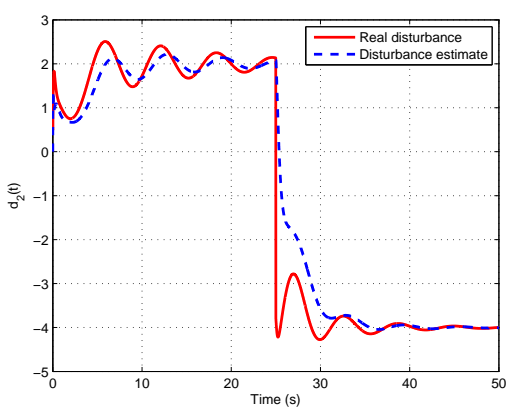

(b) $d_{2}(t)$ and $\hat{d}_{2}(t)$

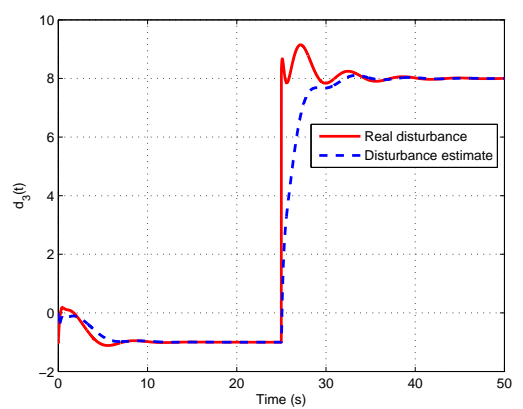

(c) $d_{3}(t)$ and $\hat{d}_{2}(t)$

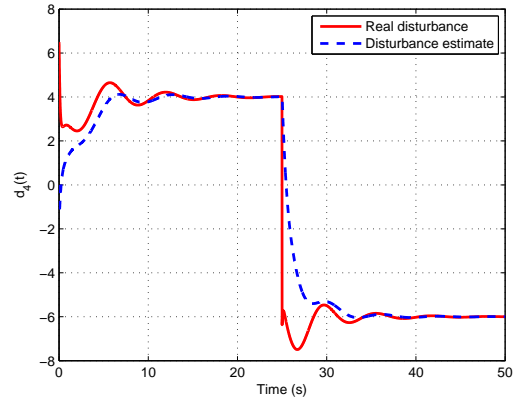

(d) $d_{4}(t)$ and $\hat{d}_{4}(t)$

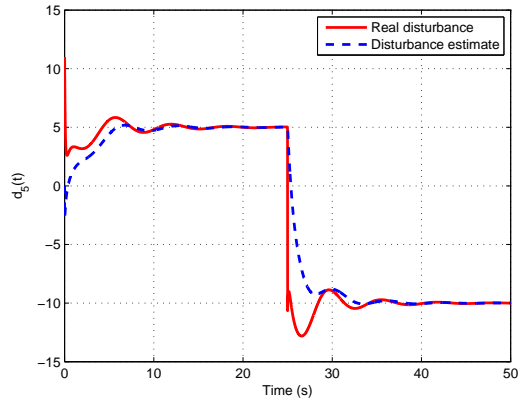

(e) $d_{5}(t)$ and $\hat{d}_{5}(t)$

Fig. 3. Disturbances and their estimates

[4] W. Yu, G. Chen, W. Ren, J. Kurths, and W. X. Zheng, "Distributed higher order consensus protocols in multiagent dynamical systems," IEEE Trans. Circuits Syst. I, Reg. Papers, vol. 58, no. 8, pp. 1924-1932, 2011.

[5] W. Ren and R. W. Beard, Distributed Consensus in Multi-Vehicle Cooperative Control. New York: Springer, 2008.

[6] G. Wen, Z. Duan, W. Yu, and G. Chen, "Consensus of multi-agent systems with nonlinear dynamics and sampled-data information: a delayed-input approach," Int. J. Robust Nonlinear Control, vol. 23, no. 6, pp. 602-619, 2013.

[7] Y. Feng, S. Xu, and B. Zhang, "Group consensus control for double-integrator dynamic multiagent?systems with fixed communication topology," Int. J. Robust Nonlinear Control, vol. 24, no. 3, pp. 532-547, 2014.

[8] Y. Liu and Y. Jia, "Consensus problem of high-order multi-agent systems with external disturbances: An $H_{\infty}$ analysis approach," Int. J. Robust Nonlinear Control, vol. 20, no. 14, pp. 1579-1593, 2010.

[9] Z. Tang, T. Huang, J. Shao, and J. Hu, "Leader-following consensus for multi-agent systems via sampled-data control," IET Control Theory and Applications, vol. 5, no. 14, pp. 1658-1665, 2011.

[10] Y. Hong, G. Chen, and L. Bushnell, "Distributed observers design for leader-following control of multi-agent networks," Automatica, vol. 44, no. 3, pp. 846-850, 2008.

[11] Z. Li, Z. Duan, G. Chen, and L. Huang, "Consensus of multiagent systems and synchronization of complex networks: A unified viewpoint," IEEE Trans. Circuits and Systems-I:Regular Papers, vol. 57, no. 1, pp. 213-224, 2010.

[12] L. Cheng, Z.-G. Hou, M. Tan, Y. Lin, and W. Zhang, "Solving a modified consensus problem of linear multi-agent systems," Automatica, vol. 47, no. 10, pp. 2218-2223, 2011.

[13] W. Ni, L. Xiao, and X. Chun, "Consensus controllability, observability and robust design for leader-following linear multi-agent system," Automatica, vol. 49, no. 7, pp. 2099-2105, 2013.

[14] H. Zhang, F. Lewis, and A. Das, "Optimal design for synchronization of cooperative systems: State feedback, observer and output feedback," IEEE Trans. Autom. Control, vol. 56, no. 8, pp. 1948-1952, 2013. 
[15] Z. Li, W. Ren, X. Liu, and L. Xie, "Distributed consensus of linear multi-agent systems with adaptive dynamic protocols," Automatica, vol. 49, no. 7, pp. 1986-1995, 2013.

[16] Z. Li, X. Liu, W. Ren, and L. Xie, "Distributed tracking control for linear multiagent systems with a leader of bounded unknown input," IEEE Trans. Autom. Control, vol. 58, no. 2, pp. 518-523, 2013.

[17] P. Lin, Y. Jia, and L. Li, "Distributed robust $H_{\infty}$ consensus control in directed networks of agents with time-delay," Systems \& Control Letters, vol. 57, no. 8, pp. 643-653, 2008.

[18] M. Andreasson, D. V. Dimarogonas, H. Sandberg, and K. H. Johansson, "Distributed control of networked dynamical systems: Static feedback, integral action and consensus," IEEE Trans. Autom. Control, vol. 59, no. 7, pp. 1750-1764, 2014.

[19] H. Yang, Z. Zhang, and S. Zhang, "Consensus of second-order multi-agent systems with exogenous disturbances," Int. J. Robust. Nonlinear Control, vol. 21, no. 9, pp. 945-956, 2010.

[20] Z. Li, Z. Duan, and F. L. Lewis, "Distributed robust consensus control of multi-agent systems with heterogeneous matching uncertainties," Automatica, vol. 50, no. 3, pp. 883-889, 2014.

[21] S. Li, Y. Jun, W. Chen, and X. Chen, "Generalized extended state observer based control for systems with mismatched uncertainties," IEEE Trans. Ind. Electron, vol. 59, no. 12, pp. 4792-4802, 2012.

[22] J. Han, "From PID to active disturbance rejection control," IEEE Trans. Ind. Electron., vol. 56, no. 3, pp. 900-906,, 2009.

[23] W. Ren and Y. Cao, Distributed Coordination of Multi-agent Networks. New York: Springer, 2011.

[24] W. Ni and D. Cheng, "Leader-following consensus of multi-agent systems under fixed and switching topologies," Systems and Control Letters, vol. 59, no. 3, pp. 209-217, 2010

[25] S. Li, J. Yang, W.-H. Chen, and X. Chen, "Generalized extended state observer based control for systems with mismatched uncertainties," IEEE Trans. Industrial Electronics, vol. 59, no. 12, pp. 4792-4802, 2012. 


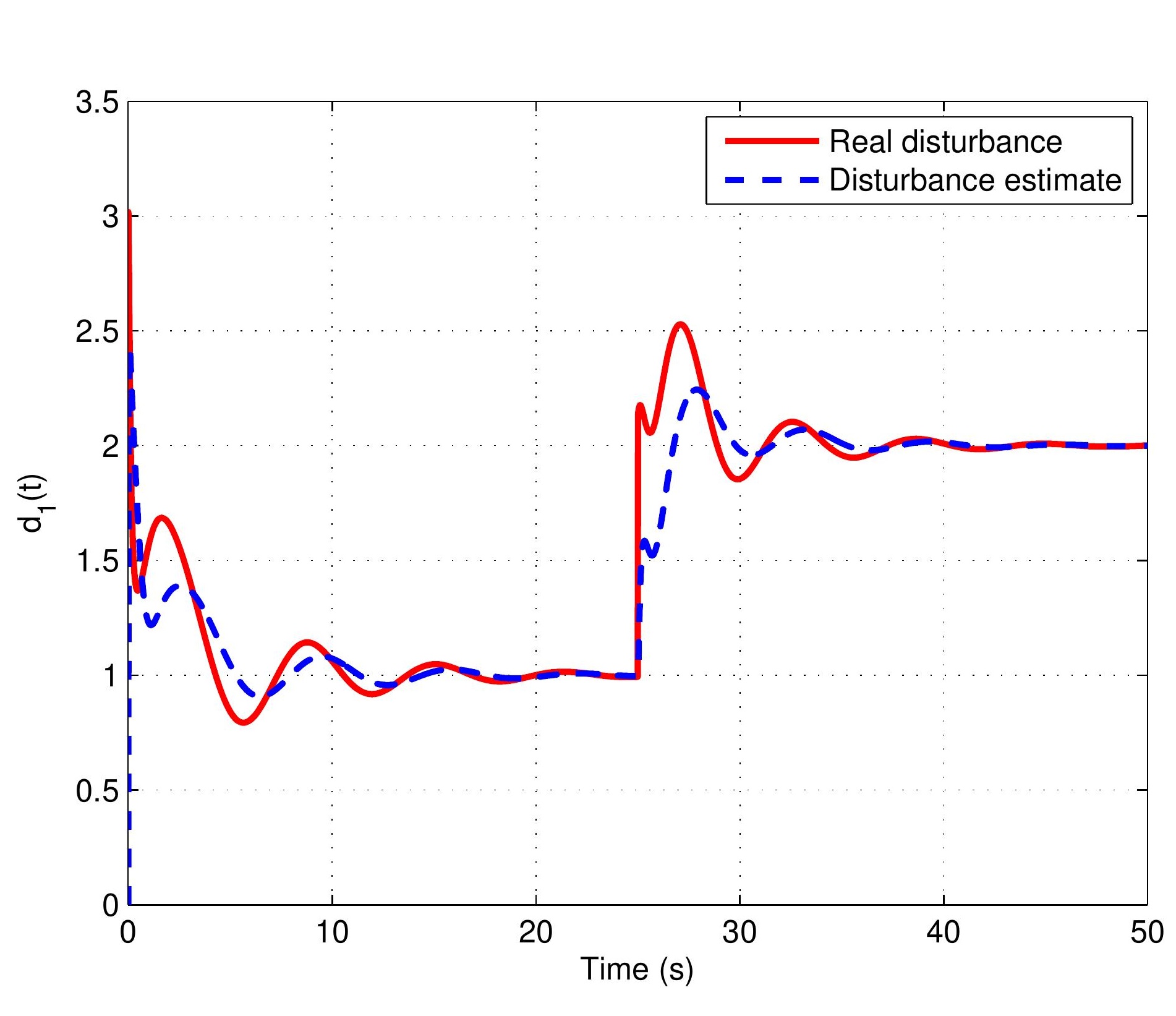

Figure

\section{Fure}




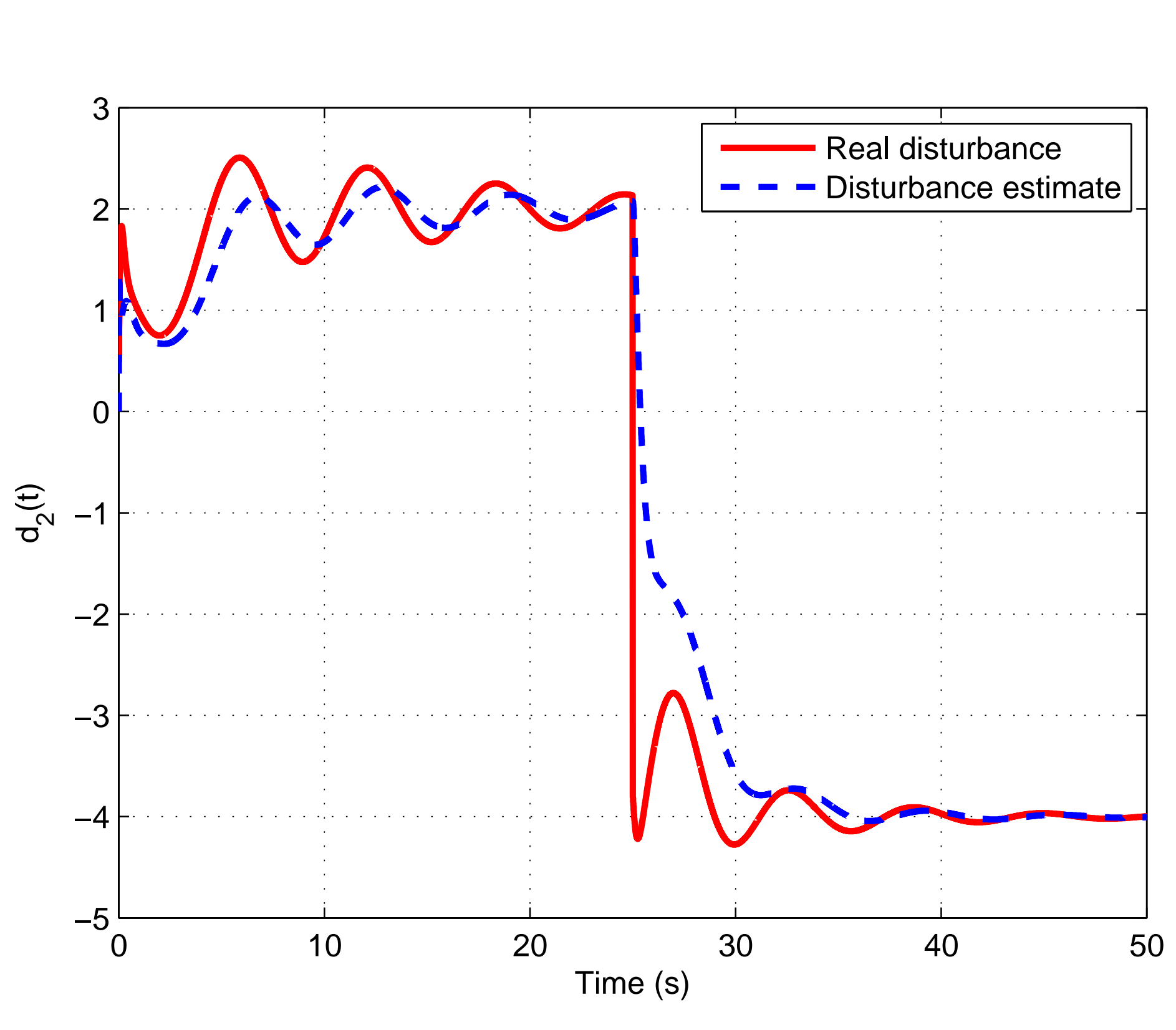

Figure

\section{.}




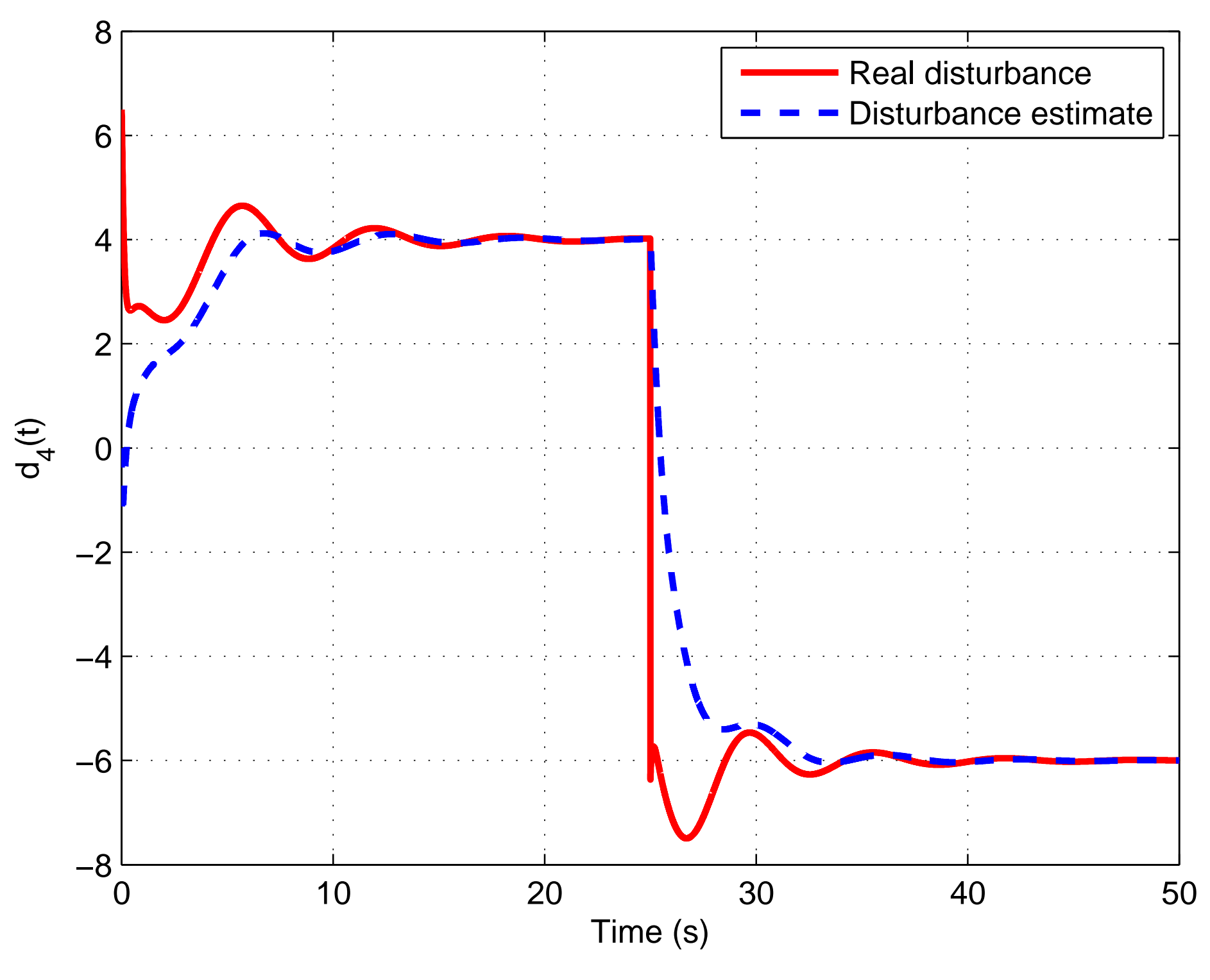

Figure 


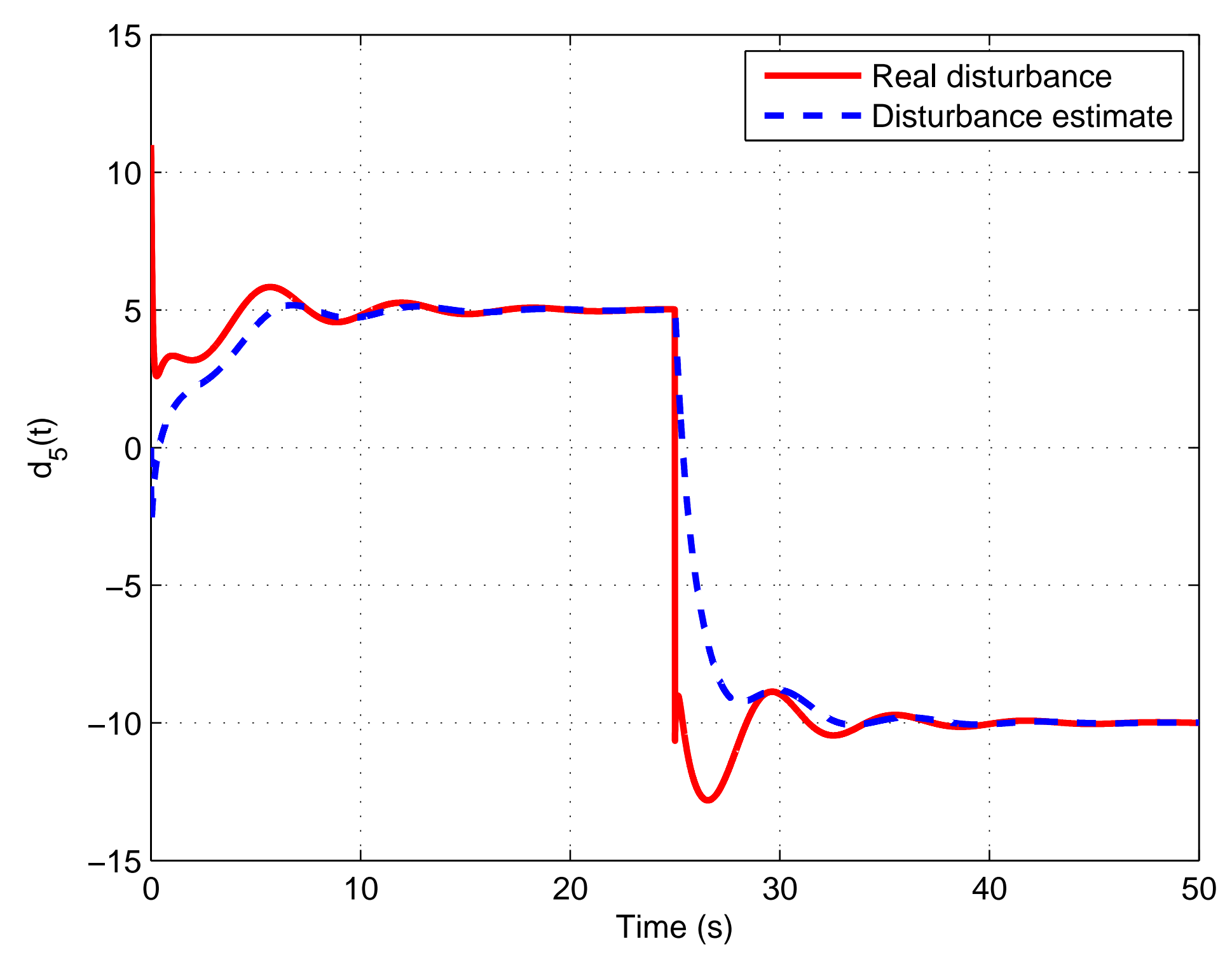

Figure 


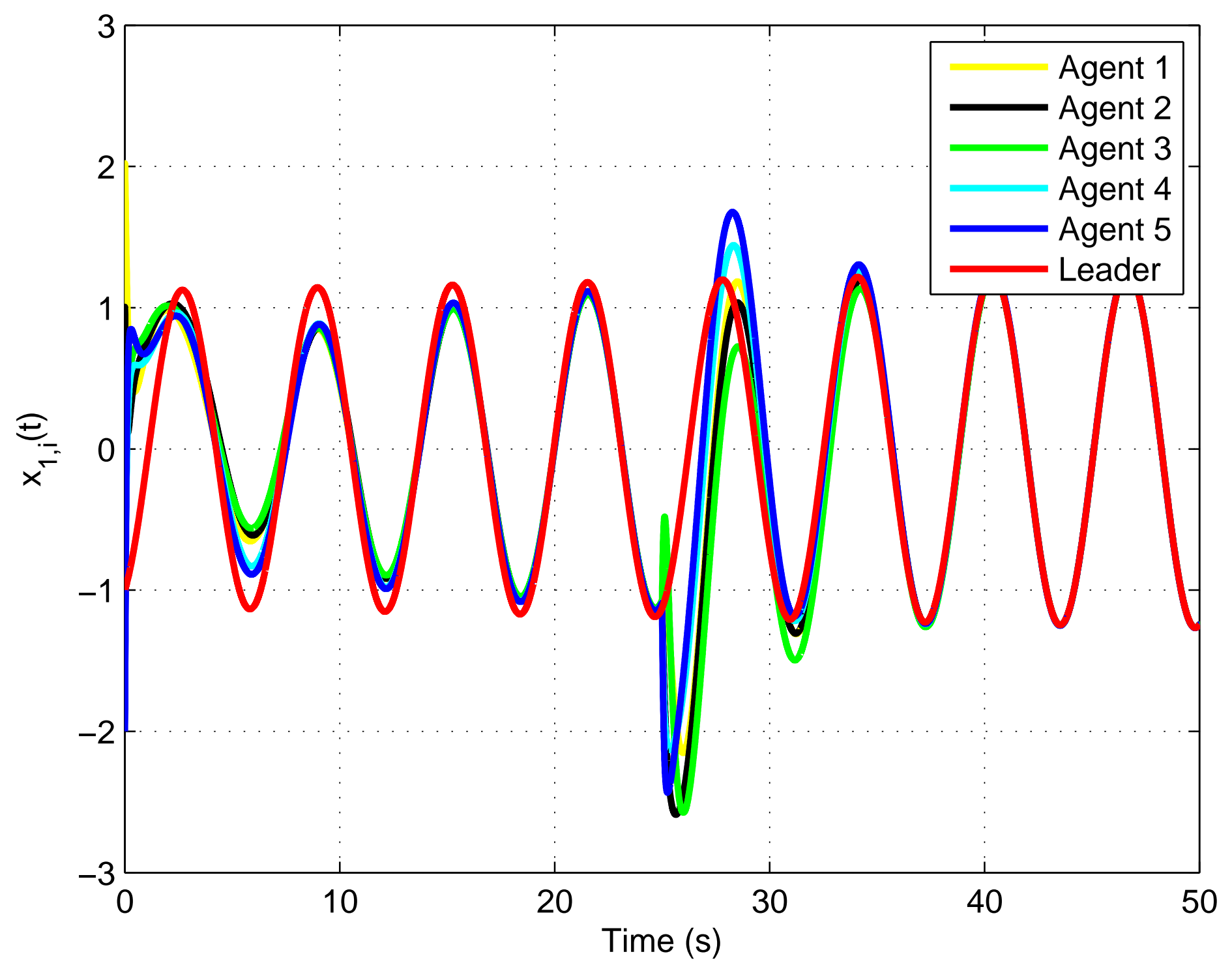




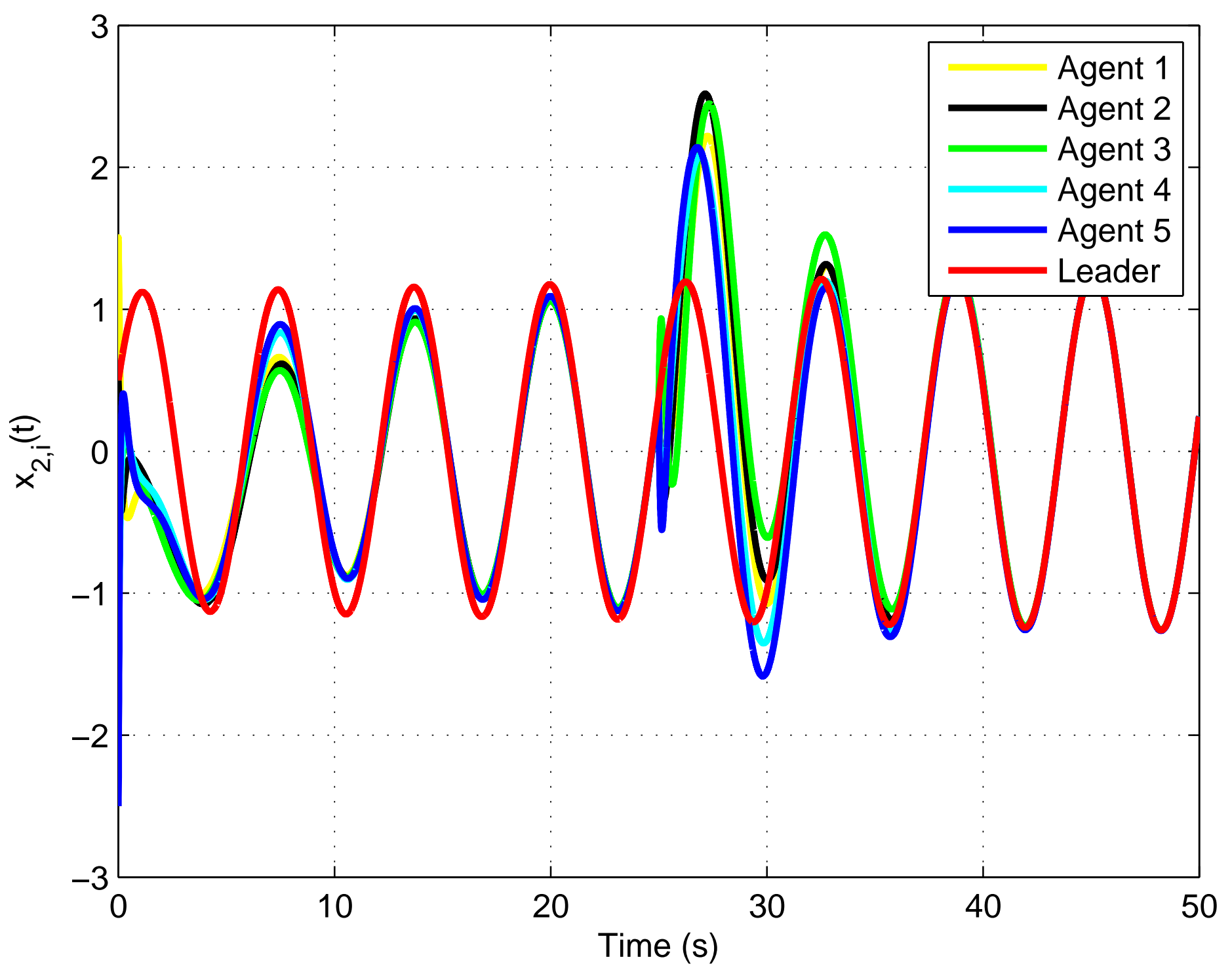




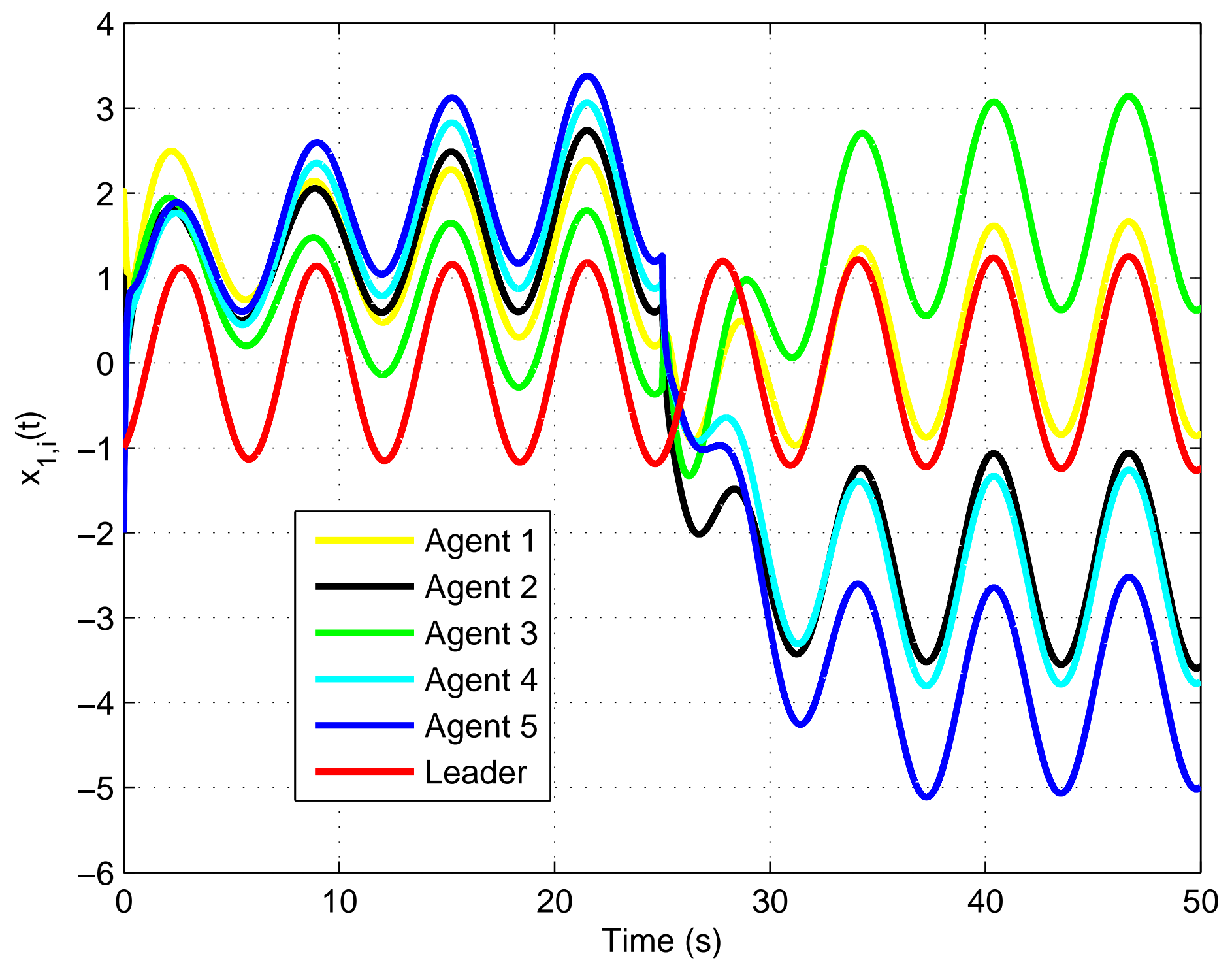




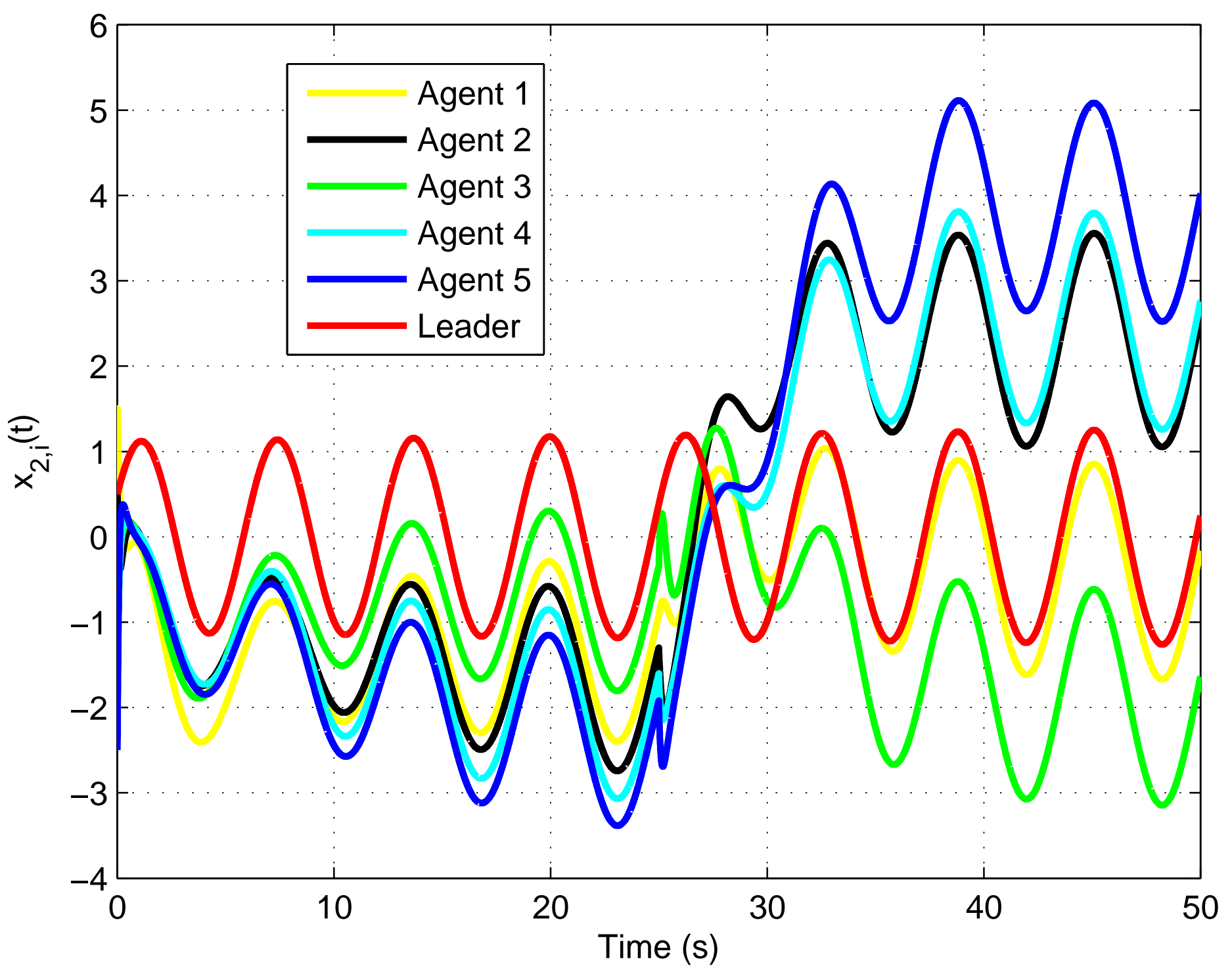


Figure

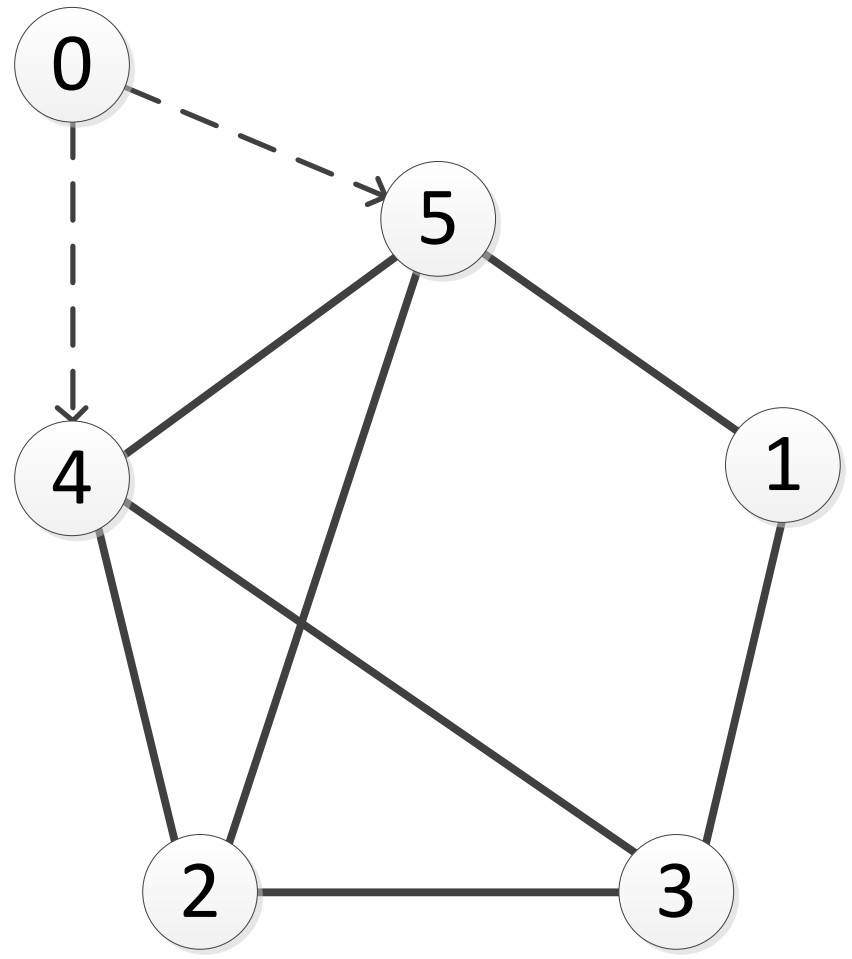




\section{${ }^{*}$ Abstract}

This paper addresses the leader-follower consensus tracking problem for multi-agent systems with identical general linear dynamics and unknown external disturbances. First, a distributed extended state observer is proposed, where both the local states and disturbance of each agent are estimated simultaneously by using the relative output information between neighbors. Then a consensus algorithm is proposed for each agent based on the relative estimated states between neighbors and its own disturbance estimate. It is shown that, with the proposed observer-based consensus algorithm, the leader-follower consensus problem can be solved. Finally, we present a simulation example to demonstrate the effectiveness of the proposed algorithm. 